\title{
Enhancing the Data Transmission Capability on Optical Fiber Communication Link Using Distinct Dispersion Compensation Technique
}

\author{
Rajat Paliwal \\ Assistant Professor, Department of Electronics and Telecommunication, Watumull Institute of Electronics \\ Engineering and Computer Technology/ University of Mumbai, India
}

\begin{abstract}
Explosive growth of broadband multimedia applications, such as high-definition television (HDTV), video on demand (VOD) and interactive games imposed a huge demand for bandwidth on the access network. The potential bandwidth of optical communication system is driving force behind the worldwide development and deployment of light wave system but dispersion limits the system performance in terms of high speed optical communication. In this paper different dispersion compensation methods are discussed, which are based on Chirped Fiber Grating (CFG), Dispersion Compensation Fibers (DCF), Self Phase Modulation (SPM) resulting from Kerr nonlinearity to overcome pulse broadening effect of Group Velocity Dispersion (GVD) which eventually have direct implication on the data transmission capability on optical fiber communication link
\end{abstract}

Keywords: Fiber Bragg Grating(FBG), Dispersion compensation Fiber (DCF), Group Velocity Dispersion $(G V D)$, Self Phase Modulation(SPM), Erbium Doped Fiber Amplifier (EDFA), Pulse Mode Dispersion (PMD), Non-Linear Schrodinger Equation(NSE)

\section{Introduction}

Access networks faced several challenges in recent years. One of these challenges is the convergence of voice, data and television (TV) on the same physical infrastructure. Moreover, explosive growth of broadband multimedia applications, such as high-definition television (HDTV), video on demand (VOD) and interactive games imposed a huge demand for bandwidth on the access network. The potential bandwidth of optical communication system is driving force behind the worldwide development and deployment of light wave system. For this reason, optical fiber became a candidate to access networks, allowing high transmission rates when compared with copper cable and coaxial cable or even with wireless communication systems.

Optical fibers can exist in different materials: glass optical fiber (GOF) and plastic optical fiber (POF). GOF is used in telecommunications window wavelength $(1310,1490$ and $1550 \mathrm{~nm})$ while the POF operates in the visible spectrum [1]. High transmission rate can be achieved by using GOF in fiber optic communication. Like other_communication system optical communication also faces problem like dispersion, attenuation and non-linear effects that lead to deterioration in its performance. The use of erbium doped fiber amplifiers (EDFA) in optical communication system has made chromatic dispersion the most significant limitation for the transmission performance since EDFAs compensate for transmission losses. The dispersion affects the system the most and it is tougher to overcome. Thus it is important to work out effective dispersion compensation methods.In this paper different dispersion compensation methods are discussed, which are based on Chirped Fiber Grating (CFG), Dispersion Compensation Fibers (DCF) and Self Phase Modulation (SPM) resulting from Kerr nonlinearity to overcome pulse broadening effect of Group Velocity Dispersion (GVD).The rest of paper is organized as follows. In section 2, various types of dispersion and its effects on data rates transmitted in optical communication system is discussed. In section 3, Chirped Fiber Grating (CFG) is presented. In section 4, Dispersion Compensation Fibers (DCF) is discussed. In section 5, SPM resulting from Kerr nonlinearity and GVD is presented. Finally section 6, concludes the paper.

\section{Dispersion}

When light signal is transmitted over long span of optical fiber, the power of signal disperses with respect to time which results in the widening of shape of pulse with time. This phenomenon is called Dispersion of signal. There are various types of dispersion $[1,2,3]$ as mentioned below :

2.1 Intramodal Dispersion- This dispersion is within mode because of finite bandwidth of signal. Broadening is due to separation of different carrier within spectral width of source and each carrier travels with different velocity inside pulse. 
2.2 Intermodal Dispersion-This dispersion is found in multi-mode fiber due to multipath propagation of different light rays and rays do not reach other end of fiber at same time.

2.3 Chromatic Dispersion- In optical fiber different colors of light travels with different speed. Even though all colors start to travel at same time but they reach destination at different times. This time delay is called as Chromatic dispersion. Here chromatic stresses the fact that's the time delay depend on color difference or wavelength difference between light and this results light pulse broadening. Chromatic dispersion is sum of Material dispersion and Waveguide dispersion.

2.3.1 Material Dispersion- The refractive index of silica which is used to make optical fiber is frequency dependent. Different frequency component or different wavelength of light travel at different speed in silica and this give rise to a phenomena called material dispersion. This dispersion cannot be changed as it depends on material refractive index which is fixed for glass.

Quantitatively it can be calculated as:

$D_{\text {MAT }}=-\lambda / c\left(d^{2} n / d \lambda^{2}\right)$ where

$\lambda=$ wavelength,

$\mathrm{n}=$ refractive index of material

$\mathrm{c}=\mathrm{velocity}$ of light

2.3.2 Waveguide dispersion: This dispersion is due to modal nature inside optical fiber. Light pulse travel partly in core and partly in cladding. Light pulse travel faster in core and slower in cladding. Over all speed depends on proportional power that is distributed in core and cladding. However power distribution is function of wavelength; longer wavelength more will be the power, hence different color of light travel at different speed because of different power distribution in core and cladding. This phenomenon is called waveguide dispersion .We can change fiber refractive index profile so waveguide dispersion compensate for material dispersion and we get zero chromatic dispersion at 1310nm wavelength [1]. (Fig. 1 taken from [8] )

Quantitatively waveguide dispersion can be calculated form B-V plot by relation:

$D_{\mathrm{WG}}=-\left(\mathrm{n}_{2} \Delta / \mathrm{c}\right)(\mathrm{V} / \lambda)\left[\mathrm{d}^{2} \mathrm{BV} / \mathrm{dV}^{2}\right]$

$\Delta=\mathrm{n}_{1}-\mathrm{n}_{2} / \mathrm{n}_{1}$

where

$\mathrm{n}_{1}=$ core refractive index

$\mathrm{n}_{2}=$ cladding refractive index

$\mathrm{B}=$ normalized propagation constant

$\mathrm{V}=$ Normalized frequency or $\mathrm{V}$ number

$\lambda=$ wavelength.

2.4 Polarized Mode Dispersion: It means different travelling speed of two different polarization mode of light pulse, so they arrive at different time which creates time delay between two polarization modes. This time delay is called differential group delay (DGD). Due to this DGD there is dispersion called Polarized mode dispersion (PMD).

Due to the dispersion, light wave travelling inside fiber gets broadened. Due to broadening of pulse, two consecutive pulse overlap with each other, causing Inter symbol interface (ISI). Due to ISI, the receiver is unable to distinguish between two symbols. This lead to error in symbol detection, so there is necessity of dispersion compensation as dispersion limits the data rates which could be sent on optical fiber for optical communication. There are various methods for dispersion compensation which are based on Chirped Fiber Grating (CFG), Dispersion Compensation Fibers (DCF), Self Phase Modulation (SPM), resulting from Kerr nonlinearity to overcome pulse broadening effect of Group Velocity Dispersion (GVD).

$1.31 \mu \mathrm{m}$ Zero-Dispersion in Step-Index SM Fiber

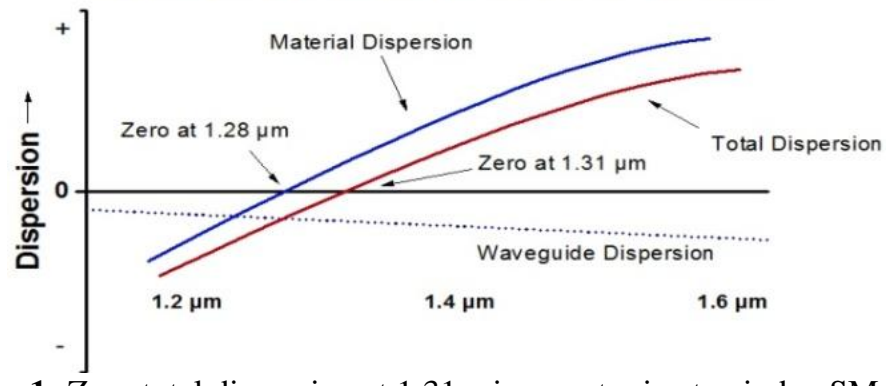

Figure 1: Zero total dispersion at 1.31 micro meter in step index SM fiber. 


\section{Chirped Fiber Grating (CFG)}

FBG is a dynamic dispersion compensator [4,5]. The FBG device is able to compensate chromatic dispersion of multiple variation of wavelength, so it is preferred solution for chromatic dispersion compensation. FBG is a reflective device composed of optical fiber that contain a modulation of its core refractive index over certain length or periodic variations of refractive index within the propagating medium (Fig2). Grating reflects light propagating through the fiber when its wavelength corresponds to modulation periodicity. The reflecting wavelength : $\left(\lambda_{B}\right)$ is called Bragg wavelength, and defined by the relationship [4,5]:

Increasing interval "chirped" FBG compensation for chromatic dispersion

$\lambda_{\mathrm{B}}=2 \mathrm{n}_{\mathrm{eff}} \Lambda_{\mathrm{G}}$

Grating period $=\Lambda_{\mathrm{G}}$

where

$\lambda_{\mathrm{B}}=$ Bragg wavelength

$\mathrm{n}_{\mathrm{eff}}$ Effective refractive index

1) Uniform Fiber Bragg Grating

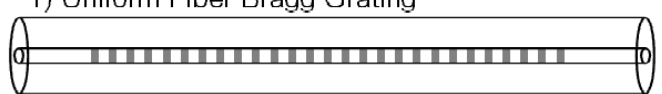

Figure 2: Uniform FBG. (taken from [5] )

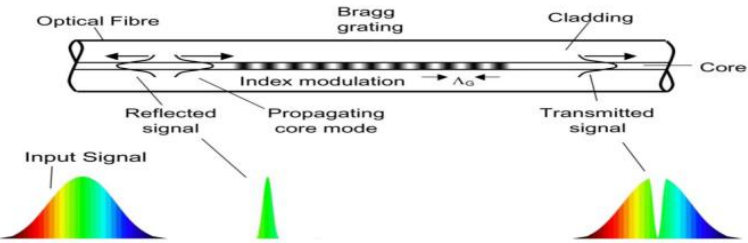

Figure 3:Uniform FBG reflects one wavelength.( taken from [5] )

FBG regular interval pattern is reflective at one wavelength (Fig 3).

Application in notch filter, add /drop multiplexer

Chirped FBG is based on principle of diffraction grating [4,5]. Chirp FBG enables the grating to reflect the various wavelengths at different points along its grating length. Therefore it sets off different delay for all different frequency or wavelength. The shorter wavelength travel faster, will arrive at FBG earlier and get reflected further up the FBG where its Bragg condition is met. Hence a longer delay is introduced for shorter wavelength which travels faster and smaller delay is introduced for larger wavelength which travels slower. Thus at the circulator the pulse will be undispersed (Fig 4).

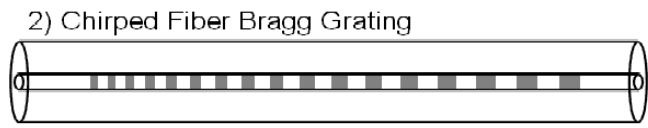

Figure 4: Chirped FBG (taken from [5] )

Here Grating period ${ }_{\left(\Lambda_{\mathrm{G}}\right)}$ is not constant but changes over length and bragg wavelength also varies along grating length. Thus different frequency components of incident optical pulse are reflected at different points depending on where Bragg condition is satisfied locally.

FBG is used in two configurations for dispersion compensation [6]:

Pre -compensation-FBG is placed at beginning of fiber network and before amplifier Post-compensation- FBG is placed at end of fiber span and before last amplifier.

\section{Dispersion Compensation Fiber (Dcf)}

This is special type of fiber having large negative dispersion of -70 to $-90 \mathrm{Ps} / \mathrm{nm} \mathrm{km}$ [6], so it can be used to compensate for positive dispersion of transmission fiber._Effects like Group velocity dispersion (GVD) and Kerr nonlinearity, degrades performance of optical WDM system. Because of non linear nature of propagation, system performance depends on power level of input of different type of fibers, on position of DCF and on amount of dispersion.

According to relative position of DCF and single mode fiber pre-compensation, post-compensation, symmetric/mix compensation is proposed [3,5,7] (Fig.5; taken from [3] ). 
Pre-compensation - In this compensation scheme, the dispersion compensating fiber of negative dispersion is placed before the standard fiber to compensate positive dispersion of standard fiber.

Post-compensation - In this compensation scheme, the dispersion compensating fiber of negative dispersion is placed after the standard fiber to compensate positive dispersion of standard fiber

Symmetric/mix-compensation-In this compensation scheme, the dispersion compensating fiber of negative dispersion is placed before and after the standard fiber to compensate positive dispersion of standard fiber.

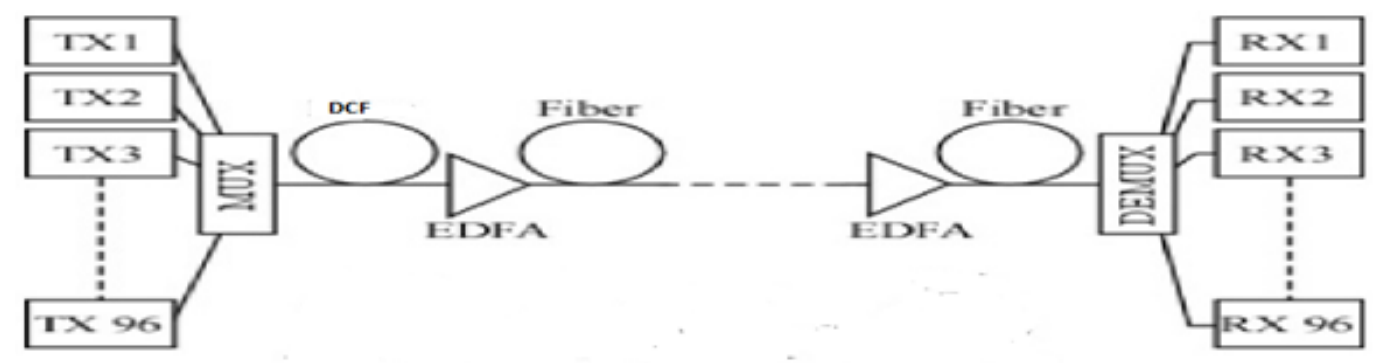

Pre-compensation scheme
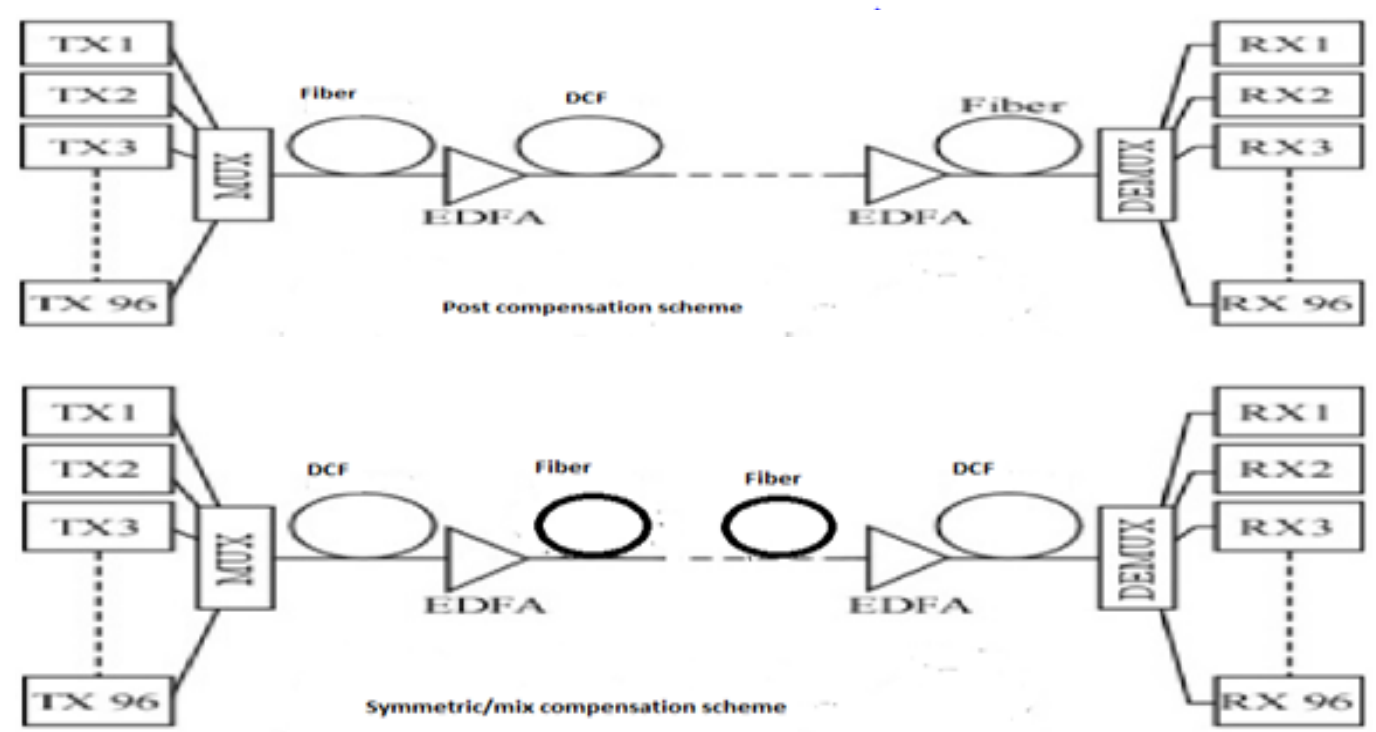

Figure 5: Different types of DCF

\section{Non Linearity Effect}

Non linearity of optical fiber means that medium properties are modified by pulse itself. Considering Kerr nonlinearity where refractive index of medium change is proportional to power density of incident light pulse inside optical fiber the refractive index of glass in presence of non linear effects [8] is given as:

$\mathrm{n}\left(\omega,|\mathrm{E}|^{2}\right)=\mathrm{n}(\underline{\omega})+\mathrm{n}_{2}|\mathrm{E}|^{2}$

where

$\mathrm{n}(\omega)=$ Linear refractive index

$\mathrm{n}_{2}|\mathrm{E}|^{2}=$ term contributing to non linearity in refractive index

$\mathrm{n}_{2}=$ non linearity coefficient

Thus the approximated differential equation which is called Non Linear Schrodinger equation(NLS) governing the behavior of pulse evolution of an optical fiber inside optical fiber in presence of non linear effect is given by [9]:

$\partial \mathrm{A} / \partial \mathrm{Z}-\mathrm{j} \beta_{2} / 2 \partial^{2} \mathrm{~A} / \partial \mathrm{t}^{2}+\mathrm{A} \alpha / 2=-\mathrm{j} \Upsilon|\mathrm{A}|^{2} \mathrm{~A}-------N o n$ Linear Schrodinger Equation

This equation consist of dispersion term $\left(j \beta_{2} / 2 \partial^{2} \mathrm{~A} / \partial \mathrm{t}^{2}\right)$, Attenuation term $(\mathrm{A} \alpha / 2)$, Non linearity term $\left(\mathrm{j} Y|\mathrm{~A}|^{2} \mathrm{~A}\right)$. In this equation:

$\mathrm{A}=$ represent envelop function

$\alpha=$ represent attenuation or loss

$\beta_{2}=$ phase constant

$\mathrm{Y}=$ non linearity parameter $=\mathrm{n}_{2} \omega_{0} / \mathrm{c} \mathrm{A}_{\mathrm{Eff}}$ 
$A_{\text {Eff }}=$ Effective area over which light is confined in optical fiber

For getting solution of above NSE equation certain approximations were done and characterizing dispersion length $\left(\mathrm{L}_{\mathrm{D}}\right)$ non linearity length $\left(\mathrm{L}_{\mathrm{NL}}\right)$ and fiber length $(\mathrm{L})$ four possible cases of solution of NLS arise which are given as

Case1: $\mathrm{L} \quad \ll \mathrm{L}_{\mathrm{D}} \quad \mathrm{L} \ll \mathrm{L}_{\mathrm{NL}^{-}}$In this case chosen length $\mathrm{L}$ of optical fiber just behaves like simple medium to transport light form one point to another without any modification in pulse characteristics.

Case2: $\mathrm{L}>>\mathrm{L}_{\mathrm{D}} \mathrm{L}<<\mathrm{L}_{\mathrm{NL}^{-}}$This regime is called group velocity limited (GVD) regime where dispersion length is very small compared to physical length of pulse but due to low power level in pulse non linearity effect is not present.

Case 3: $\mathrm{L}\left\langle<\mathrm{L}_{\mathrm{D}} \mathrm{L}\right\rangle>\mathrm{L}_{\mathrm{NL}^{-}}$This regime is called non linearity limited regime where dispersion length is very large but power in pulse is sufficient enough to show linearity effect. In this case self phase modulation (SPM) is observed due to change in refractive index of material by pulse shape itself.

Case4: $\mathrm{L} \quad<<\mathrm{L}_{\mathrm{D}} \quad \mathrm{L}<<\mathrm{L}_{\mathrm{NL}}$-This case arise when pulse width is narrow and having high pulse power. In this situation both dispersion and non linear effects co-exist and we see very special kind of pulse propagation called solitonic propagation where:

$\mathrm{L}_{\mathrm{D}}=\mathrm{T}_{0}^{2} /\left|\beta_{2}\right|$
$\mathrm{L}_{\mathrm{NL}}=1 / \mathrm{YP}$
$\mathrm{P}=$ power

GVD Regime: In this case (Case2), amplitude spectrum of pulse remain unchanged and only component with different frequency undergo phase change which is proportional to $(\omega)^{2}$ and distance $Z$. Frequency chirp phenomena is also observed where inside pulse frequency is going to change from one end of pulse to another end of pulse. Here in plot two chirp phenomena are defined; one is normal dispersion region $\left(\beta_{2}>0, \lambda<1300 \mathrm{~nm}\right)$ where chirp is positive and second is anamolous dispersion regime $\left(\beta_{2}<0, \lambda>1300 \mathrm{~nm}\right)$ where chirp is negative (Fig 6 :taken form Nptel)

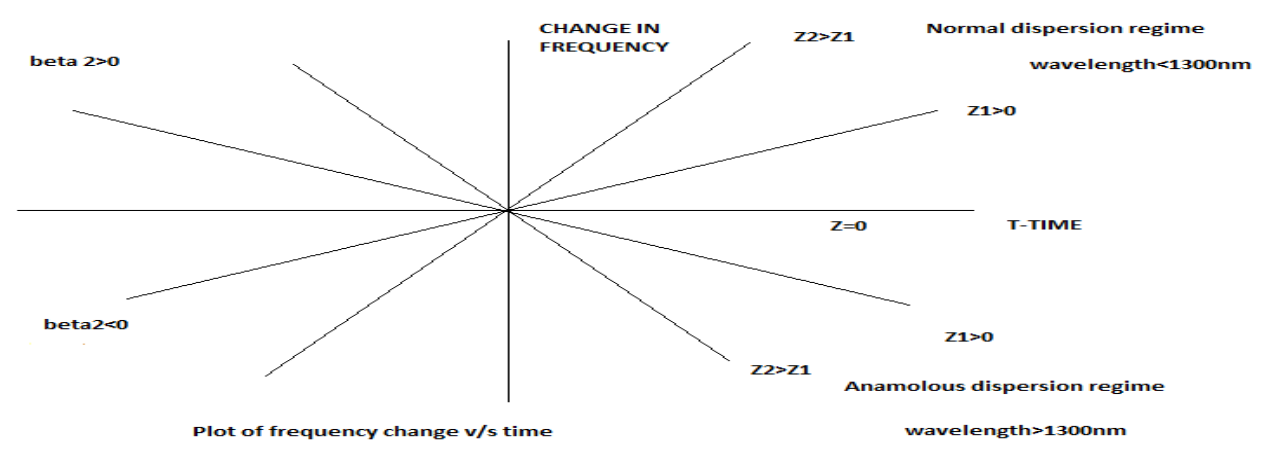

Figure6: Frequency change with time

Non linearity limited regime: In this case (Case3), SPM effect is seen but frequency chirp is positive. Pulse in time domain remain intact and only phase changes, so frequency spectrum gets modified (Fig.7).

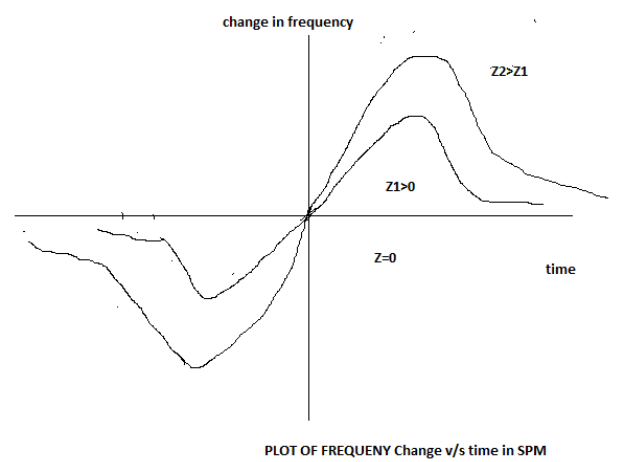

Figure 7: Frequency change with time in SPM.( taken form Nptel )

If we consider Case 4 and choose parameter of pulse in such a way that dispersion and nonlinearity both play a role and the frequency chirp created by anomalous dispersion in GVD regime which is negative is balanced by positive slope of frequency chirp created by SPM effect, then both chirp phenomena will cancel each other and we will get 
constant frequency all over the pulse and then there will be no pulse broadening in time domain [9].This phenomena of balancing effect of frequency chirp to compensate the dispersion using nonlinear effect give rise to pulse which can propagate without broadening or contracting over longer distance provided non linear effects are maintained on optical fiber. This phenomena where two effects balance each other such that pulse do not get distorted over longer distance is called Soliton.

We do normalization of parameters so above NSE equation [9] is modified as:

$\partial \mathrm{U} / \partial \mathcal{E}-\mathrm{j} \operatorname{sgn}\left(\beta_{2}\right) 1 / 2 \partial^{2} \mathrm{U} / \partial \mathrm{G}^{2}+\mathrm{j} \mathrm{N}^{2}|\mathrm{U}|^{2} \mathrm{U}=0$---Non Linear Schrodinger Equation with normalized parameters (8)

Normalized amplitude $\mathrm{U}=\mathrm{A} / \mathrm{P}_{0} \quad$ Normalized distance $\varepsilon=\mathrm{Z} / \mathrm{L}_{\mathrm{D}}$

Normalized to pulse width $\mathrm{T}_{0}$

$\mathrm{T}=\mathrm{T} / \mathrm{T}_{0}$

$\mathrm{N}=$ order of soliton $=\mathrm{L}_{\mathrm{D}} / \mathrm{L}_{\mathrm{NL}} \quad[10]$

Solution of above equation NSE with certain approximations give rise to soliton and for N=1 we get fundamental soliton (Fig 8) given by [8,9]:

$\mathrm{U}(\varepsilon, \mathrm{G})=\operatorname{Sech}(\mathrm{G}) \mathrm{e}^{-\mathrm{j} \varepsilon / 2}---$ Equation of fundamental Soliton
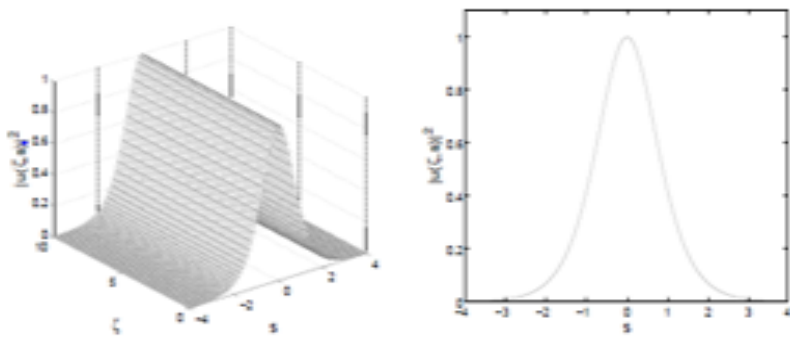

Figure8: Fundamental Soliton

\section{Conclusion}

We have discussed various dispersion compensation techniques which include chirped FBG pre and post compensation methods, DCF pre, post and mix compensation scheme and Kerr nonlinearity effect. I conclude by reducing dispersion on optical fiber by various methods we can enhance the performance of optical fiber communication link and allow transmission at high speed in range of few tens of Giga bits per second or more by choosing proper parameters.

\section{Acknowledgement}

The author sincerely acknowledge contributions of Dr. Bharat Paliwal (M.D. Anaesthesiology) and Dr. Vinita Paliwal (B.P.T.,PGDY) for their guidance and support in preparing this article.

\section{References}

[1]. M.G..Patel and S. B. Khant, SolitonTransmission in Fiber Optics for Long Distance Communication, International Journal of Advanced Research in Electrical, Electronics and Instrumentation Engineering(2), 2014, 7100-7107.

[2]. N.K.Khalon and G.Kaur, Various Dispersion Compensation Techniques for Optical System:ASurvey, Open journal of communication and software (1), 2014, 64-73.

[3]. R. Rout, S. Pradhan and S.Patnaik, Role of DCF technique for enhancing optical fiber communication System utility, International Research Journal of Engineering and Technology, 2(7), 2015, 691-696.

[4]. K. Kumar, A.K.Jaiswal, M. Kumar and N. Agarwal, Performance Analysis of dispersion compensating Fiber Bragg Grating (FBG) in Optical Communication, International Journal of Current Engineering and Technology, 4(3), 2014, $1527-1531$.

[5]. A.J. Agarwal, M. Kumar and R. Saxena,Comparison of different techniques of dispersion compensation, International Journal of Electronics and Computer Science Engineering, 2(3), 2013, 912-918.

[6]. M. Kaur, H. Sarangal and P.Bagga, Dispersion compensation with Dispersion Compensating Fibers (DCF),International Journal of Advanced Research in Computer and Communication Engineering 4(2), 2015, 354-356.

[7]. G. Singh, J. Saxena and G. Kaur, Dispersion compensation Using FBG and DCF in 120 Gbps WDM System, International Journal of Engineering and Innovative Technology, 3(6), 2014, 514-518.

[8]. S.Mundhe, S.Bhosale and S. Shirodkar, Evolution of Solitons in Optical Communication, International Journal of Research in Advent Technology, (3), 2015, 1-4.

[9]. R.Gangwar, S.P Singh, and N.Singh, Soliton based optical communication, Progress In Electromagnetics Research, (74), 2007, 157166.

[10]. L.Bohac, TheSoliton Transmissions in Optical Fibers, Information And Communication Tecnologies and Services, 8(5), 2010, 107111. 